\title{
Impact of clinical-pathological factors on locoregional recurrence in mastectomy patients with T1-2N1 breast cancer: who can omit adjuvant radiotherapy?
}

\section{Xiaofang Wang}

Fudan University Shanghai Cancer Center

Xiaomeng Zhang

Fudan University Shanghai Cancer Center Jurui Luo

Fudan University Shanghai Cancer Center

\section{Li Zhang}

Fudan University Shanghai Cancer Center

\section{Xingxing Chen}

Fudan University Shanghai Cancer Center

\section{Zhaozhi Yang}

Fudan University Shanghai Cancer Center

\section{Xin Mei}

Fudan University Shanghai Cancer Center

\section{Xiaoli Yu}

Fudan University Shanghai Cancer Center

\section{Zhen Zhang}

Fudan University Shanghai Cancer Center

\section{Zhimin Shao}

Fudan University Shanghai Cancer Center

\section{Xiaomao Guo}

Fudan University Shanghai Cancer Center Jinli Ma ( $\square$ Ma-Jinli@outlook.com )

Fudan University Shanghai Cancer Center https://orcid.org/0000-0001-6127-648X

\section{Research Article}

Keywords: Breast cancer, Early stage, PMRT, Prognostic factors

Posted Date: May 5th, 2021 
DOI: https://doi.org/10.21203/rs.3.rs-450920/v1

License: (c) (1) This work is licensed under a Creative Commons Attribution 4.0 International License. Read Full License 


\section{Abstract}

Purpose: Postmastectomy radiation therapy (PMRT) in T1-T2 tumors with 1-3 positive axillary lymph nodes (ALNs) is controversial. This study was to identify prognostic factors of locoregional control (LRC) following mastectomy with or without PMRT for patients with T1-2N1 breast cancer and to discuss the selection of patients who might omit PMRT.

Materials and Methods: Between January 2006 and December 2010, the data of 1474 postmastectomy patients staged PT1-2N1 were analyzed. PMRT was applied in 663 patients. LRC and disease-free survival (DFS) were calculated using the Kaplan-Meier method. Cox regression model was applied in the univariate and multivariate analyses to recognize the recurrence risk factors.

Results: With the median follow-up duration of 93 months (range, 5-168 months), 78 patients (5.3\%) failed to secure LRC and 220 patients (14.9\%) experienced any recurrence. The 7.7-year LRC and DFS was $94.9 \%$ and $85.4 \%$ respectively in the entire cohort. PMRT significantly improved 7.7-year LRC from $93.4 \%$ to $96.6 \%(p=0.005)$, but not the DFS ( $p=0.335)$. Multivariate analysis revealed that PMRT was an independent prognostic factor of LRC $(p<0.001)$, meanwhile, age $\leq 40$ years $(p=0.012)$, histological grade $3(p=0.004), 2-3$ positive nodes $(p<0.001)$ and tumor size of $3-5 \mathrm{~cm}(p=0.045)$ were significantly associated with decreased LRC. The 7.7-year LRC for patients with 0,1 , and 2-4 risk factors was $97.7 \%$ / 98.9\% ( $P=0.233), 95.3 \%$ / 98.0\% ( $P=0.092)$, and 80.3\% / 94.8\% $(P<0.001)$ in the non-PMRT and PMRT group respectively.

Conclusion: In patients with T1-2N1 breast cancer, clinical-pathological factors such as young age, histological grade 3, 2-3 positive nodes, and tumor size of 3-5cm were identified to be predictors of a poorer LRC following mastectomy. Patients with 0-1 risk factor might consider the omission of PMRT.

\section{Introduction}

Postmastectomy radiotherapy (PMRT) has long been the standard for patients with tumors larger than $5 \mathrm{~cm}$ or with 4 or more positive axillary lymph nodes (ALNs). However, for early-staged patients with T1-2 tumors and 1-3 positive ALNs, the role of PMRT remains controversial. The use of PMRT is supported by the 15-year result of EORTC 22922/10925 [1] and the 20-year result of the Early Breast Cancer Trialists' Collaborative Group (EBCTCG) meta-analysis [2], which showed significant reductions of locoregional recurrence (LRR) and breast cancer mortality by PMRT. Based on these, the NCCN guideline strongly recommends the locoregional irradiation in patients staged pT1-2N1 in recent years [3]. However, some concerns were evoked from today's perspective: patients who participated were not all staged at pT1-2N1; novel systemic treatment was not available at the time of some trials conducted; in terms of toxicity, the application of novel radiation techniques is expected to further reduce radiation-associated heart disease. So as proposed by ASTRO and St. Gallen Consensus [4, 5], PMRT should be conducted individually in consideration of risk factors and toxicity in the early-staged patients. 
Previous studies have recognized several risk factors, such as patient age, tumor size, number and ratio of positive lymph nodes, molecular subtype, and lymph-vascular invasion (LVI) [6-9]. However, how to stratify patients into different risk groups was not well defined. The purpose of this retrospective study was to make PMRT decision recommendations according to risk stratification in the era of modern medicine.

\section{Methods}

\section{Patients}

Of all breast cancer patients diagnosed at Fudan University Shanghai cancer center between January 2006 and December 2012, 1621 female patients underwent mastectomy and were staged pT1-2N1. The medical records were extracted from the computerized database. Patients who had contralateral advanced breast cancer $(n=9)$, or with neoadjuvant systemic therapy $(n=57)$, or with follow-up time shorter than 3 months $(n=81)$ were excluded. The final cohort included 1474 patients for retrospective analysis. The review of data was approved by the Ethical Committee and Institutional Review Board of our center.

The clinical-pathological information of eligible patients was collected, including age at diagnosis, laterality, tumor location, tumor histology, histological grade, tumor size, number of positive and examined lymph nodes, LVI, and estrogen receptor (ER), progesterone receptor (PR), human epidermal receptor 2 (HER2) status. ER and PR status were evaluated by immunohistochemistry (IHC). A cutoff value of $1 \%$ was used to dichotomize cases into positive and negative [10]. Hormonal receptor (HR) + was defined as ER+ and/or PR+, and HR- as both ER- and PR-. HER2 status was determined by IHC as well. Tumors were considered HER2 positive if they scored $3+$, indeterminate if $2+$, and negative if $1+$ or 0 on IHC. When IHC was indeterminate, tumors were considered HER2 positive with amplification (ratio >= 2.0) by fluorescence in situ hybridization (FISH) analysis [11].

\section{Treatment}

All patients underwent mastectomy with negative surgical margins and axillary dissection. Following surgery, adjuvant chemotherapy was given according to international guidelines. HR-positive patients received adjuvant endocrine therapy. HER2 positive patients were given anti-HER2 therapy.

Following chemotherapy, PMRT was administered at the discretion of treating physician. Generally, a dose of 50Gy in 25 fractions was delivered to the ipsilateral chest wall (CW) for all patients, and the regional nodes for node-positive patients, which included supraclavicular (SCV) and infraclavicular (ICV) with or without internal mammary nodes (IMN), using 3D forward planning field-in-field or simplified inverse-planning intensity-modulated RT technique.

\section{Endpoints}


Follow-up data was last updated on Oct 31,2020. The primary endpoint of this study was locoregional control (LRC), defined as clinical, radiographic, or pathological evidence of LRR within ipsilateral CW and/or regional nodes (i.e., ipsilateral ALN, SCV, ICV, or IMN). Recurrences at other sites except for localregional were considered distant metastases (DM). The secondary endpoint was disease-free survival (DFS), measured from the date of surgery to the time of first LRR, DM, death, or the last visit.

\section{Statistical analysis}

Patient characteristics between PMRT and non-PMRT subgroups were compared using the Pearson's $\chi 2$. The probabilities of LRC and DFS were calculated using the Kaplan-Meier method and compared between groups using the log-rank test. Recurrence risk factors were recognized using Cox regression model in the univariate and multivariate analyses, and subsequently used to stratify patients into low-, intermediate-, and high-risk groups. The level of significance was set at $p<0.05$ (two-sided), using SPSS 26.0.

\section{Results}

\section{Patient Characteristics}

Among the 1474 patients analyzed, the median age was 51 years (range, 23-86 years). 1418 patients $(96.2 \%)$ were diagnosed with invasive ductal carcinoma. The median invasive tumor size was $2.3 \mathrm{~cm}$ (range, $0.1-5 \mathrm{~cm})$. The primary tumor staging was $\mathrm{T} 1$ in $45.3 \%$ of patients $(\mathrm{n}=668)$, and $\mathrm{T} 2$ in $54.7 \%$ of patients $(n=806)$. The median number of ALNs examined was 17 (range, 10-39); the percentage of patients having 1,2 , and 3 positive lymph nodes was $52.2 \%(n=770), 28.9 \%(n=426)$, and $18.9 \%(n=$ $278)$, respectively. On IHC staining, HR was positive in $79.5 \%$ of patients $(n=1172)$. HER2 was positive in $20.5 \%$ of patients $(n=302)$.

Adjuvant chemotherapy was administered in $93.1 \%$ of patients $(n=1373)$. The most common regimen was the combination of anthracycline and taxane $(935 ; 68.1 \%)$, followed by anthracycline-based or taxane-based regimens. About $97.8 \%$ of patients completed $4-8$ cycles of adjuvant chemotherapy before radiotherapy (RT). Adjuvant endocrine therapy was administered in $95.1 \%(n=1115)$ of HR-positive patients, and anti-HER2 therapy was in $47.0 \%(n=142)$ of HER2-positive patients.

PMRT was applied in $45.0 \%$ of patients $(n=663)$. Of them, 592 patients received irradiation to both CW and regional lymph nodes, 1 patient received irradiation to $\mathrm{CW}$ only, and the rest received irradiation as well, while irradiated sites were not well described in the medical record.

Table 1 compared patients' clinical and treatment characteristics between PMRT and non-PMRT subgroups. Patients associated with high risk factors were more likely to be directed to PMRT, including young age, high histological grade, LVI+, larger tumor, advanced lymph nodes, and HER2-positive subtype.

\section{Recurrence and Survival Outcomes}


With the median follow-up duration of 93 months (range, 5-168 months), a total of 78 patients (5.3\%) developed LRR. Overall, the most common recurrence site was regional nodes alone (55.1\%), followed by CW alone (32.1\%). Of regional recurrences, the most common site was SCV/ICV, followed by IMN. Table 2 compared the anatomical distribution of LRR between PMRT and non-PMRT subgroups, but the chisquare test did not show a statistically significant difference $(\chi 2=2.54, p=0.281)$. For the entire cohort, the 7.7-year cumulative LRC was $94.9 \%$. PMRT significantly improved 7.7 -year LRC from $93.4 \%$ to $96.6 \%$ $(p=0.005)$ (Fig. 1a).

By the date of the last follow-up, 220 (14.9\%) patients experienced any recurrence. Among these, 209 patients had DM, including 67 patients with concomitant LRR; and 11 patients had isolated LRR. Table 3 compared the recurrence patterns between PMRT and non-PMRT subgroups, and the difference was statistically significant $(\chi 2=7.652, p=0.022)$. For the entire cohort, the actuarial 7.7-year DFS was $85.4 \%$. No statistically significant difference was observed between the non-PMRT and PMRT subgroups for the 7.7-year DFS interval (84.2\% vs. $86.7 \%, p=0.335$ ) (Fig. 1b).

\section{Prognostic Risk Factors}

The correlation of LRC and DFS with various prognostic factors is shown in Table 4. In univariate analysis, multiple factors including age, histological grade, number of positive ALNs, and PMRT were significantly associated with LRC. With the exception of PMRT, these factors along with tumor size were also significantly associated with DFS. Triple-negative breast cancer was associated with worse LRC and DFS in comparison with other molecular subtypes, however, the difference was not statistically significant. Additionally, subgroup analysis showed that the application of anti-HER2 therapy could improve both LRC $(p=0.024)$ and DFS $(p<0.001)$ in HER2-positive patients, and the use of endocrine therapy could improve DFS $(p=0.015)$ in HR-positive population.

Advanced multivariate analysis confirmed that clinical-pathological factors including younger age of $\leq$ 40 years (adjusted hazard ratio [HR], 2.02; 95\% confidence interval [Cl], 1.17-3.50; $p=0.012$ ), tumor size of $3-5 \mathrm{~cm}(\mathrm{HR}, 1.73 ; 95 \% \mathrm{Cl}, 1.01-2.97 ; \mathrm{p}=0.045)$, histological grade $3(\mathrm{HR}, 1.97 ; 95 \% \mathrm{Cl}, 1.24-3.12 ; \mathrm{p}=$ 0.004), 2-3 positive ALNs (HR, 2.46; $95 \% \mathrm{Cl}, 1.51-3.99 ; \mathrm{p}<0.001)$, and no PMRT delivery (HR, 3.36; $95 \%$ $\mathrm{Cl}, 2.11-6.14 ; \mathrm{p}<0.001)$ were significantly associated with a poorer LRC. Besides the newly identified factor of PMRT (HR, 1.63; 95\% Cl, 1.22-2.18; $p=0.001)$, younger age of $\leq 40$ years, tumor size of $3-5 \mathrm{~cm}$, histological grade 3 , and 2-3 positive ALNs remained independent predictors of a shorter DFS interval (Table 5).

\section{Outcomes of Risk Groups}

In total, 1398 patients were stratified into three groups by recurrence risk, including 377 patients (27.0\%) in low-risk group (no risk factor), 572 patients (40.9\%) in intermediate-risk group (1 risk factor), and 449 patients $(32.1 \%)$ in high-risk group (2-4 risk factors). 
Figure 2 presents the Kaplan-Meier analyses of LRR and DFS stratified by recurrence risk. The 7.7-year LRC for patients in low-, intermediate-, and high-risk group was $97.7 \%$ / 98.9\% ( $P=0.233), 95.3 \%$ / 98.0\% $(P=0.092)$, and $80.3 \% / 94.8 \%(P<0.001)$ in the non-PMRT and PMRT subgroups, respectively.

Meanwhile, PMRT was significantly associated with a longer DFS time in high-risk patients, with 7.7-year DFS of $66.6 \%$ in non-PMRT subgroup and $80.5 \%$ in PMRT subgroup $(P=0.002)$. However, no benefit from PMRT was observed in low-risk $(P=0.309)$ and intermediate-risk $(P=0.388)$ patients.

\section{Discussion}

Our study analyzes the LRC and survival outcomes among 1474 breast cancer patients who underwent mastectomy and were pathologically staged T1-2N1, and makes PMRT recommendations according to risk stratification. The result demonstrated a 7.7-year LRC of $94.9 \%$ and DFS of $85.4 \%$ for the entire cohort in the era of modern therapy. Besides, PMRT was proved to be an independent predictor of LRC and DFS, despite that more patients in the PMRT subgroup had unfavorable disease features, such as larger tumor size, 2-3 positive ALNs, LVI+, and HER2-positive subtype. However, the benefit of PMRT was not statistically significant for the 7.7-year DFS in the entire population $(p=0.335)$.

Evidence indicating that PMRT improves outcomes of patients with pT1-2N1 disease spans two treatment eras. The EBCTCG firstly powerfully proved the value of PMRT for node-positive patients in a meta-analysis including individual data of 8135 women from 22 trials during the period of $1964-86$. The subgroup analysis of 1314 pN1 patients substantiated a reduction of 10 -year LRR from $20.3 \%$ to $3.8 \%$, 10-year overall recurrence from $45.7 \%$ to $34.2 \%$, and 20 -year breast-cancer mortality from $50.2 \%$ to $42.3 \%$ with the application of PMRT [2]. Another proof is the subgroup analysis of DBCG $82 \mathrm{~b} \& \mathrm{c}$ trial conducted between 1982 - 90. In the analysis of 1152 pN1 patients, PMRT reduced the 15-year locoregional failure (LRF) rate from $27 \%$ to $4 \%$ and improved the 15 -year survival from $48 \%$ to $57 \%$ [12]. However, for patients since 1996, lower LRF rates were observed due to newer systemic treatment, modern RT techniques, and meticulous attention to surgical margins. In accordance with our data, recent studies reported that the estimated 5- and 10-year LRCs were $91 \%$ - 97\% and $89 \%$ - 95\% without PMRT, respectively [13-19], which have an improvement compared with historic studies mentioned above. Therefore, several prospective and retrospective studies explored the value of PMRT in the era of modern therapy. The recently published EORTC 22922/10925 trial, which included 4004 patients, with 23.9\% receiving mastectomy and 43.1\% having 1-3 positive lymph nodes, revealed that the addition of regional nodes irradiation significantly reduced 15-year recurrence and breast cancer mortality [1]. Besides, in the subgroup analysis of BCIRG-005, PMRT improved 10-year LRC from 91\% to 98\% ( $p=0.001)$ in pT1-2N1 patients [18]. Luo C et al reported that PMRT was significantly associated with decreased LRR (5-year cumulative incidence $1.6 \%$ vs. $6.0 \% ; \mathrm{HR}, 0.248 ; 95 \% \mathrm{Cl}, 0.121-0.509 ; \mathrm{P}<0.001$ ) in early-staged patients who underwent mastectomy [20]. So together with our findings, these results confirmed the value of locoregional irradiation among pT1-2N1 patients in the modern treatment era.

Given such favorable LRC even in non-PMRT patients with pT1-2N1 breast cancer, a key clinical challenge is to determine which patients can omit PMRT. In this study, patients were stratified by risk factors such 
as young age, tumor size of 3-5cm, 2-3 positive ALNs, and high histological grade, which were recognized in the univariate and multivariate analysis. For high-risk patients ( $\geq 2$ risk factors), PMRT significantly improved both LRC $(p<0.001)$ and DFS $(p=0.002)$. In the intermediate-risk group (1 risk factor), a trend toward improvement of LRC ( $p=0.092)$ but not DFS $(p=0.388)$ was observed with the addition of PMRT. However, no benefit from PMRT was observed in low-risk patients (0 risk factor), with 7.7-year LRC of $98.9 \% / 97.7 \%(p=0.233)$ and DFS of $93.0 \% / 89.5 \%(p=0.309)$ in the PMRT and non-PMRT patients, respectively. The outcomes of our study were consistent with previous studies with similar designs. Park $\mathrm{HJ}$ et al conducted a multicenter analysis of 1382 patients staged pT1-2N1 in Korea (KROG 14-23), and identified that age $<35$ years, T2 stage, high tumor grade, close resection margin, triple-negative biological subtype, and 2-3 positive nodes were independent risk factors of LRR. Further analysis revealed that patients with $0-1,2-3$, and $4-6$ risk factors owned the 5 -year LRR of $3.6 \%, 7.5 \%$, and $12.7 \%$, respectively, and demonstrated that patients with 2 or more risk factors might benefit from PMRT [9]. Luo CX et al included tumor size, number of positive nodes, ER status, histologic grade, and LVI status in a nomogram for predicting LRR in a cohort of 1141 cases, and found that PMRT was significantly correlated with decreased LRR only in the high-risk group (5-year LRR $2.2 \%$ vs. $14.9 \%, p<0.001$ ); the 5year LRR was relatively low in the low-risk $(0.4 \%)$ and intermediate-risk $(6.1 \%)$ non-PMRT group, and no survival advantage was observed in these two risk groups [20].

Unfavorable molecular subtypes including triple-negative and HER2-positive breast cancer are considered to be predictors of poor outcomes in many studies. Our analysis failed to recognize these biologic subtypes to statistically significant predictors of poor LRC and DFS. The possible reason might be the improvement of patient's survival with the application of novel systemic therapy; in our study, almost all patients received 4-8 cycles of chemotherapy in triple-negative $(97.4 \% ; 154 / 158)$ and HER2-positive $(99.2 \% ; 119 / 120)$ subtypes. Unfortunately, only $47.0 \%$ (142/302) of HER2-overexpressed patients received anti-HER2 therapy for economic reasons. The subgroup analysis revealed that the use of antiHER2 drugs significantly improved both LRC $(p=0.024)$ and DFS time $(p<0.001)$, as reported in other studies [21, 22]. In this study, no more than $5 \%$ of HR-positive patients did not receive adjuvant endocrine therapy because of PR positive only or ER weakly positive that didn't meet the positive criteria eight years ago. In the HR-positive subtype, we confirmed that the application of endocrine drugs was associated with a longer DFS interval $(p=0.121)$, but not a better LRC $(p=0.015)$.

Our study still has certain limitations, including its retrospective nature, limited follow-up time, and loss of individual data. To reduce the deviation of data missing, we conducted the univariate analysis firstly before the multivariate analysis and thus maximum reduced data deletion in the calculating process. Despite such limitations, our study offers LRC and DFS estimates and makes PMRT recommendations according to risk stratification in the era of modern medicine. The retrospective-prospective study of NSABP B-14 and B-20 revealed that recurrence score (RS) of 21-gene OncotypeDX was efficient in predicting LRR, which leads to an era of precision medicine [23]. Ongoing trials in patients with T1-2 breast cancer and 1 to 3 positive nodes are expected to provide us more evidence of adjuvant RT. Of these, CCTG MA39 (TAILOR RT) trial will help to clarify the feasibility of omitting RT in low-risk patients with the assessment of RS. 


\section{Conclusions}

This study demonstrates favorable LRC and DFS in patients who underwent mastectomy and were staged pT1-2N1 in the era of modern systemic therapy. On multivariate analysis, factors associated with increased recurrence risk include young age, tumor size of $3-5 \mathrm{~cm}$, histological grade 3 , and 2-3 positive lymph nodes. Stratifying by these factors, the risk group was significantly associated with LRR risk and RT benefit. Low- to intermediate-risk patients had a small benefit from PMRT and might consider omitting PMRT; High-risk patients had a greater benefit and therefore should consider the routine use of PMRT. Further prospective investigations are needed to improve risk stratification and estimates of RT benefit in individuals with $\mathrm{pT} 1-2 \mathrm{~N} 1$ breast cancer after mastectomy.

\section{Abbreviations}

\begin{tabular}{ll} 
PMRT & postmastectomy radiotherapy \\
\hline ALNs & axillary lymph nodes \\
\hline EBCTCG & the Early Breast Cancer Trialists' Collaborative Group \\
\hline LRR & locoregional recurrence \\
\hline LVI & lymph-vascular invasion \\
\hline ER & estrogen receptor \\
\hline PR & progesterone receptor \\
\hline HER2 & human epidermal receptor 2 \\
\hline IHC & immunohistochemistry \\
\hline CW & ipsilateral chest wall \\
\hline SCV & supraclavicular \\
\hline ICV & infraclavicular \\
\hline IMN & internal mammary nodes \\
\hline LRC & locoregional control \\
\hline DM & distant metastases \\
\hline DFS & disease-free survival \\
\hline RT & radiotherapy \\
\hline LRF & locoregional failure \\
\hline RS & recurrence score \\
\hline
\end{tabular}




\section{Declarations}

\section{Funding}

This work was supported by Xiaomao Guo and the National Natural Science Foundation of China (Grant Number. 81872457).

\section{Conflict of interest}

The authors declare no conflicts of interest.

\section{Availability of data and material}

The datasets generated during and/or analysed during the current study are available from the corresponding author on reasonable request.

\section{Code availability}

Not applicable.

\section{Authors' contributions}

Conception and design: Jinli Ma and Xiaomao Guo

Collection and assembly of data: Xiaofang Wang

Data analysis and interpretation: Xiaofang Wang, Jinli Ma and Xiaomao Guo

Manuscript writing: Xiaofang Wang and Jinli Ma

Manuscript revision final approval: All authors

Financial support: Xiaomao Guo

\section{Ethics approval}

The study is a retrospective analysis. Therefore, informed consent from patients was not required for this study. The review of data was approved by the Ethical Committee and Institutional Review Board of Fudan University Shanghai Cancer Center.

\section{Consent to participate}

Not applicable.

\section{Consent for publication}

Written informed consent for publication was obtained from all participants. 


\section{References}

1. Poortmans PM, Weltens $C$, Fortpied $C$ et al (2020) Internal mammary and medial supraclavicular lymph node chain irradiation in stage I-III breast cancer (EORTC 22922/10925): 15-year results of a randomised, phase 3 trial. Lancet Oncol 21(12): 1602-1610. https://doi.org/10.1016/S14702045(20)30472-1

2. Ebctcg, McGale P, Taylor $C$ et al (2014) Effect of radiotherapy after mastectomy and axillary surgery on 10-year recurrence and 20-year breast cancer mortality: meta-analysis of individual patient data for 8135 women in 22 randomised trials. Lancet 383(9935): 2127-2135. https://doi.org/10.1016/S0140-6736(14)60488-8

3. The National Comprehensive Cancer Network. NCCN clinical practice guidelines in oncology breast cancer. https://www.nccn.org/patients. Accessed 17 April 2021.

4. Curigliano G, Burstein HJ, Winer EP et al (2017). De-escalating and escalating treatments for earlystage breast cancer: the St. Gallen International Expert Consensus Conference on the Primary Therapy of Early Breast Cancer. Ann Oncol 28(8): 1700-1712. https://doi.org/10.1093/annonc/mdx308

5. Recht A, Comen EA, Fine RE et al (2016). Postmastectomy Radiotherapy: An American Society of Clinical Oncology, American Society for Radiation Oncology, and Society of Surgical Oncology Focused Guideline Update. J Clin Oncol 34(36): 4431-4442. https://doi.org/10.1200/JC0.2016.69.1188

6. Kassak F, Rossier C, Picardi C et al (2019) Postmastectomy radiotherapy in T1-2 patients with one to three positive lymph nodes - Past, present and future. Breast 48: 73-81. https://doi.org/10.1016/j.breast.2019.09.008

7. Abdel-Rahman $O$ (2019). Impact of postmastectomy radiotherapy on the outcomes of breast cancer patients with T1-2N1 disease : An individual patient data analysis of three clinical trials. Strahlenther Onkol 195(4): 297-305. https://doi.org/ 10.1007/s00066-018-1343-x

8. Kim SI, Cho SH, Lee JS et al (2013) Clinical relevance of lymph node ratio in breast cancer patients with one to three positive lymph nodes. Br J Cancer 109(5): 1165-1171. https://doi.org/10.1038/bjc.2013.465

9. Park HJ, Shin KH, Kim JH et al (2017) Incorporating Risk Factors to Identify the Indication of Postmastectomy Radiotherapy in N1 Breast Cancer Treated with Optimal Systemic Therapy: A Multicenter Analysis in Korea (KROG 14-23). Cancer Res Treat 49(3): 739-747. https://doi.org/10.4143/crt.2016.405

10. Hammond ME, Hayes DF, Dowsett M et al (2010) American Society of Clinical Oncology/College Of American Pathologists guideline recommendations for immunohistochemical testing of estrogen and progesterone receptors in breast cancer. J Clin Oncol 28(16): 2784-2795. https://doi.org/10.1200/JC0.2009.25.6529 
11. Wolff AC, Hammond MEH, Allison KH et al (2018) Human Epidermal Growth Factor Receptor 2 Testing in Breast Cancer: American Society of Clinical Oncology/College of American Pathologists Clinical Practice Guideline Focused Update. J Clin Oncol 36(20): 2105-2122. https://doi.org/10.1200/JC0.2018.77.8738

12. Overgaard $M$, Nielsen HM, Overgaard J (2007) Is the benefit of postmastectomy irradiation limited to patients with four or more positive nodes, as recommended in international consensus reports? A subgroup analysis of the DBCG 82 b\&c randomized trials. Radiother Oncol 82(3): 247-253. https://doi.org/10.1016/j.radonc.2007.02.001

13. Tendulkar RD, Rehman S, Shukla ME et al (2012). Impact of postmastectomy radiation on locoregional recurrence in breast cancer patients with 1-3 positive lymph nodes treated with modern systemic therapy. Int J Radiat Oncol Biol Phys 83(5): e577-581. https://doi.org/10.1016/j.ijrobp.2012.01.076

14. Sharma R, Bedrosian I, Lucci A et al (2010). Present-day locoregional control in patients with $\mathrm{t} 1$ or $\mathrm{t} 2$ breast cancer with 0 and 1 to 3 positive lymph nodes after mastectomy without radiotherapy. Ann Surg Oncol 17(11): 2899-2908. https://doi.org/10.1245/s10434-010-1089-x

15. Macdonald SM, Abi-Raad RF, Alm El-Din MA et al (2009) Chest wall radiotherapy: middle ground for treatment of patients with one to three positive lymph nodes after mastectomy. Int J Radiat Oncol Biol Phys 75(5): 1297-1303. https://doi.org/10.1016/j.jijrobp.2009.01.007

16. McBride A, Allen P, Woodward W et al (2014) Locoregional recurrence risk for patients with $T 1,2$ breast cancer with 1-3 positive lymph nodes treated with mastectomy and systemic treatment. Int $\mathrm{J}$ Radiat Oncol Biol Phys 89(2): 392-398. https://doi.org/10.1016/j.ijrobp.2014.02.013

17. Moo TA, McMillan R, Lee $M$ et al (2013) Selection criteria for postmastectomy radiotherapy in t1-t2 tumors with 1 to 3 positive lymph nodes. Ann Surg Oncol 20(10): 3169-3174. https://doi.org/10.1245/s10434-013-3117-0

18. Tam MM, Wu SP, Perez C et al (2017) The effect of post-mastectomy radiation in women with one to three positive nodes enrolled on the control arm of BCIRG-005 at ten year follow-up. Radiother Oncol 123(1): 10-14. https://doi.org/ 10.1016/j.radonc.2017.03.001

19. Bazan JG, Majithia L, Quick AM et al (2018) Heterogeneity in Outcomes of Pathologic T1-2N1 Breast Cancer After Mastectomy: Looking Beyond Locoregional Failure Rates. Ann Surg Oncol 25(8): 22882295. https://doi.org/10.1245/s10434-018-6565-8

20. Luo C, Zhong X, Deng L et al (2019) Nomogram Predicting Locoregional Recurrence to Assist Decision-Making of Postmastectomy Radiation Therapy in Patients With T1-2N1 Breast Cancer. Int J Radiat Oncol Biol Phys 103(4): 905-912. https://doi.org/10.1016/j.ijrobp.2018.11.005

21. Tseng YD, Uno H, Hughes ME et al (2015) Biological Subtype Predicts Risk of Locoregional Recurrence After Mastectomy and Impact of Postmastectomy Radiation in a Large National Database. Int J Radiat Oncol Biol Phys 93(3): 622-630. https://doi.org/10.1016/j.jirobp.2015.07.006

22. Cameron D, Piccart-Gebhart MJ, Gelber RD et al (2017) 11 years' follow-up of trastuzumab after adjuvant chemotherapy in HER2-positive early breast cancer: final analysis of the HERceptin 
Adjuvant (HERA) trial. Lancet 389(10075): 1195-1205. https://doi.org/10.1016/S0140-

6736(16)32616-2

23. Mamounas EP, Tang G, Fisher B et al (2010) Association between the 21-gene recurrence score assay and risk of locoregional recurrence in node-negative, estrogen receptor-positive breast cancer: results from NSABP B-14 and NSABP B-20. J Clin Oncol 28(10): 1677-1683.

https://doi.org/10.1200/JC0.2009.23.7610

\section{Tables}

Table 1: Patient characteristics and comparison between PMRT and non-PMRT subgroups.

\begin{tabular}{|c|c|c|c|c|c|}
\hline \multirow{2}{*}{ Parameters } & & \multirow{2}{*}{$\begin{array}{c}\text { total } \\
\text { No. (\%) }\end{array}$} & \multirow{2}{*}{$\begin{array}{c}\text { non-PMRT } \\
\text { No. (\%) }\end{array}$} & \multirow{2}{*}{$\begin{array}{c}\text { PMRT } \\
\text { No. (\%) }\end{array}$} & \multirow{2}{*}{$\boldsymbol{P}$} \\
\hline & & & & & \\
\hline Age & $>40$ & 1237 (83.9) & $713(87.9)$ & $524(79.0)$ & \\
\hline Laterality & Right & $716(48.6)$ & $396(48.8)$ & $320(48.3)$ & \\
\hline \multirow[t]{2}{*}{ Tumor location } & Medial & $347(23.5)$ & $167(20.6)$ & $180(27.1)$ & .003 \\
\hline & Central & $96(6.5)$ & $50(6.2)$ & $46(6.9)$ & \\
\hline \multirow[t]{4}{*}{ Histological grade } & Grade 1 & $16(1.1)$ & $9(1.2)$ & $7(1.1)$ & .004 \\
\hline & Grade 2 & $926(62.8)$ & $538(66.3)$ & $388(58.5)$ & \\
\hline & Grade 3 & $456(30.9)$ & $219(27.0)$ & $237(35.7)$ & \\
\hline & Unknow & $76(5.2)$ & 45 (5.5) & $31(4.7)$ & \\
\hline Tumor size & $53 \mathrm{~cm}$ & $1212(82.2)$ & $697(85.9)$ & $515(77.7)$ & $<.001$ \\
\hline \multirow[t]{3}{*}{ Lymph-vascular invasion } & Negative & $510(34.6)$ & $348(42.9)$ & $162(24.4)$ & $<.001$ \\
\hline & Positive & $825(56.0)$ & $354(43.6)$ & $471(71.0)$ & \\
\hline & Unknow & $139(9.4)$ & $109(13.4)$ & $31(4.5)$ & \\
\hline \multirow[t]{6}{*}{ Biologic subtype } & HR+/Her2- & $914(62.0)$ & $529(65.2)$ & $385(58.1)$ & $<.001$ \\
\hline & $\mathrm{HR}+/ \mathrm{Her} 2+$ & $182(12.3)$ & $76(9.4)$ & $106(16.0)$ & \\
\hline & HR-/Her2+ & $120(8.1)$ & $53(6.5)$ & $67(10.1)$ & \\
\hline & HR-/Her2- & $158(10.7)$ & $82(10.1)$ & $76(11.5)$ & \\
\hline & $\mathrm{HR}+/ \mathrm{Her} 2$ unkonwn & $76(5.2)$ & $53(6.5)$ & $23(3.5)$ & \\
\hline & HR-/Her2 unkonwn & $24(1.6)$ & $18(2.2)$ & $6(0.9)$ & \\
\hline \multirow[t]{2}{*}{ Adjuvant chemotherapy } & No & $101(6.9)$ & $93(11.5)$ & $8(1.2)$ & $<.001$ \\
\hline & Yes & $1373(93.1)$ & $718(88.5)$ & $655(98.8)$ & \\
\hline Chemotherapy regimen & Anthracycline & $246(17.9)$ & $184(25.6)$ & $62(9.5)$ & \\
\hline \multirow[t]{3}{*}{ Hormonal therapy (in HR+ patients) } & No & $42(3.6)$ & $23(3.5)$ & $19(3.7)$ & .018 \\
\hline & Yes & $1115(95.1)$ & $621(94,4)$ & $494(96.1)$ & \\
\hline & Unknown & $15(1.3)$ & $14(2.1)$ & $1(0.2)$ & \\
\hline \multirow[t]{2}{*}{ Anti-Her2 therapy } & No & $1327(90.0)$ & $760(93.7)$ & $567(85.5)$ & $<.001$ \\
\hline & Yes & $147(10.0)$ & $51(6.3)$ & $96(14.5)$ & \\
\hline \multirow[t]{2}{*}{ Anti-Her2 therapy (in Her $2+$ patients) } & No & $160(53.0)$ & $80(62.0)$ & $82(46.2)$ & .007 \\
\hline & Yes & $142(47.0)$ & $49(38.0)$ & $93(53.8)$ & \\
\hline
\end{tabular}

Abbreviations: PMRT = postmastectomy radiotherapy; $\mathrm{HR}=$ hormonal receptor .

Table 2: Patterns of locoregional recurrence. 


\begin{tabular}{lccccc}
\hline \multirow{2}{*}{ Parameters } & $\begin{array}{c}\text { Total } \\
(\mathbf{n}=\mathbf{7 8})\end{array}$ & & $\begin{array}{c}\text { Non-PMRT } \\
(\mathbf{n}=\mathbf{5 4})\end{array}$ & & $\begin{array}{c}\text { PMRT } \\
(\mathbf{n}=\mathbf{2 4})\end{array}$ \\
\cline { 2 - 2 } \cline { 5 - 6 } \cline { 5 - 5 } & No. (\%) & & No. (\%) & & No. (\%) \\
\hline CW alone & $25(32.1)$ & & $15(27.8)$ & & $10(41.7)$ \\
Regional nodes alone & $43(55.1)$ & & $33(61.1)$ & & $10(41.7)$ \\
$\quad$ Axilla alone & 2 & & 2 & & 0 \\
$\quad$ IMN alone & 5 & & 2 & & 3 \\
$\quad$ SCV/ICV alone & 31 & & 25 & & 6 \\
$\quad$ Multiple regions & 5 & & 4 & & 1 \\
CW + regional nodes & $10(12.8)$ & & $6(11.1)$ & & $4(16.7)$ \\
\hline
\end{tabular}

Abbreviations: $\mathrm{PMRT}=$ postmastectomy radiotherapy; $\mathrm{CW}=$ chest wall; $\mathrm{IMN}=$ internal mammary nodes; SCV = supraclavicular; ICV = infraclavicular.

Table 3: Patterns of recurrence at any site.

\begin{tabular}{|c|c|c|c|}
\hline \multirow{2}{*}{ Parameters } & $\begin{array}{c}\text { Total } \\
(\mathbf{n}=\mathbf{2 2 0})\end{array}$ & $\begin{array}{c}\text { Non-PMRT } \\
(\mathrm{n}=125)\end{array}$ & $\begin{array}{c}\text { PMRT } \\
(n=95)\end{array}$ \\
\hline & No. (\%) & No. (\%) & No. (\%) \\
\hline LRR alone & $11(5.0)$ & $8(6.4)$ & $3(3.2)$ \\
\hline DM alone & $142(64.5)$ & $71(56.8)$ & $71(74.7)$ \\
\hline LRR + DM & 67 (30.5) & $46(36.8)$ & $21(22.1)$ \\
\hline
\end{tabular}

Abbreviations: $\mathrm{PMRT}=$ postmastectomy radiotherapy; $\mathrm{LRR}=$ locoregional recurrence; $\mathrm{DM}$ $=$ distant metastasis.

Table 4: Univariate analyses of patient clinical and treatment-related factors for LRC and DFS. 


\begin{tabular}{|c|c|c|c|c|}
\hline Parameters & 7.7-year LRC (\%) & $P$ & 7.7-year DFS (\%) & $\boldsymbol{P}$ \\
\hline Age & & .032 & & .015 \\
\hline$\leq 40$ & 92.2 & & 80.8 & \\
\hline$>40$ & 95.4 & & 86.2 & \\
\hline Laterality & & .633 & & .605 \\
\hline Left & 94.5 & & 85.1 & \\
\hline Right & 95.3 & & 85.7 & \\
\hline Tumor location & & .898 & & .392 \\
\hline Medial & 94.8 & & 87.5 & \\
\hline Central & 94.8 & & 84.9 & \\
\hline Outer & 95.3 & & 83.1 & \\
\hline Histological grade & & .019 & & .004 \\
\hline Grade 1 & 100.0 & & 93.8 & \\
\hline Grade 2 & 96.0 & & 87.0 & \\
\hline Grade 3 & 92.1 & & 80.7 & \\
\hline Grade 1 versus Grade 2 & & .333 & & .308 \\
\hline Grade 2 versus Grade 3 & & .009 & & .002 \\
\hline Tumor size & & .070 & & $<.001$ \\
\hline$\leq 3$ & 95.5 & & 87.3 & \\
\hline 3-5 & 91.9 & & 76.2 & \\
\hline No. of positive nodes & & .018 & & .002 \\
\hline 1 & 96.6 & & 88.5 & \\
\hline 2 & 93.5 & & 81.6 & \\
\hline 3 & 92.2 & & 82.5 & \\
\hline 1 versus 2 & & .022 & & .001 \\
\hline 2 versus 3 & & .717 & & .617 \\
\hline Lymph-vascular invasion & & .719 & & .286 \\
\hline Negative & 94.3 & & 87.0 & \\
\hline Positive & 94.9 & & 84.4 & \\
\hline Biologic subtype & & .176 & & .413 \\
\hline $\mathrm{HR}+/ \mathrm{Her} 2-$ & 95.2 & & 86.5 & \\
\hline $\mathrm{HR}+/ \mathrm{Her} 2+$ & 95.8 & & 83.8 & \\
\hline HR-/Her2+ & 96.1 & & 87.4 & \\
\hline HR-/Her2- & 91.3 & & 81.4 & \\
\hline Adjuvant chemotherapy & & .377 & & .254 \\
\hline No & 97.7 & & 89.3 & \\
\hline Yes & 94.7 & & 85.1 & \\
\hline PMRT & & .005 & & .335 \\
\hline No & 93.4 & & 84.2 & \\
\hline Yes & 96.6 & & 86.7 & \\
\hline Hormonal therapy (in HR+ patients) & & .121 & & .015 \\
\hline No & 92.0 & & 74.3 & \\
\hline Yes & 95.4 & & 86.1 & \\
\hline Anti-Her2 therapy (in Her2+ patients) & & .024 & & $<.001$ \\
\hline No & 93.6 & & 78.3 & \\
\hline Yes & 98.4 & & 92.5 & \\
\hline
\end{tabular}

Abbreviations: $\mathrm{LRC}=$ locoregional control; $\mathrm{DFS}=$ disease-free survival; $\mathrm{PMRT}=$ postmastectomy radiotherapy; $\mathrm{HR}=$ hormonal receptor.

Table 5: Multivariate analysis of prognostic factors for outcomes of LRC and DFS. 


\begin{tabular}{|c|c|c|c|c|}
\hline \multirow{2}{*}{ Parameters } & \multicolumn{2}{|l|}{ LRC } & \multicolumn{2}{|c|}{ DFS } \\
\hline & $\operatorname{HR}(95 \% \mathrm{Cl})$ & p-value & HR $(95 \% \mathrm{Cl})$ & p-value \\
\hline Age (years) ( $\$ 40$ vs $>40)$ & $2.02(1.17-3.50)$ & .012 & $1.57(1.12-2.20)$ & .008 \\
\hline Histological grade (grade 3 vs grade $1 \sim 2$ ) & $1.97(1.24-3.12)$ & .004 & $1.55(1.17-2.04)$ & .002 \\
\hline No. of positive nodes (2 3 vs 1 ) & $2.46(1.51-3.99)$ & $<.001$ & $1.69(1.27-2.24)$ & $<.001$ \\
\hline Tumor size $(3-5 \mathrm{~cm}$ vs $0-3 \mathrm{~cm})$ & $1.73(1.01-2.97)$ & .045 & $1.91(1.40-2.59)$ & $<.001$ \\
\hline PMRT (no vs yes) & $3.36(2.11-6.14)$ & $<.001$ & $1.63(1.22-2.18)$ & .001 \\
\hline
\end{tabular}

Abbreviations: LRC $=$ locoregional control; $\mathrm{DFS}=$ disease-free survival; $\mathrm{CI}=$ confidence interval; $\mathrm{HR}=$ hazard ratio; $\mathrm{PMRT}=$ postmastectomy radiotherapy.

\section{Figures}
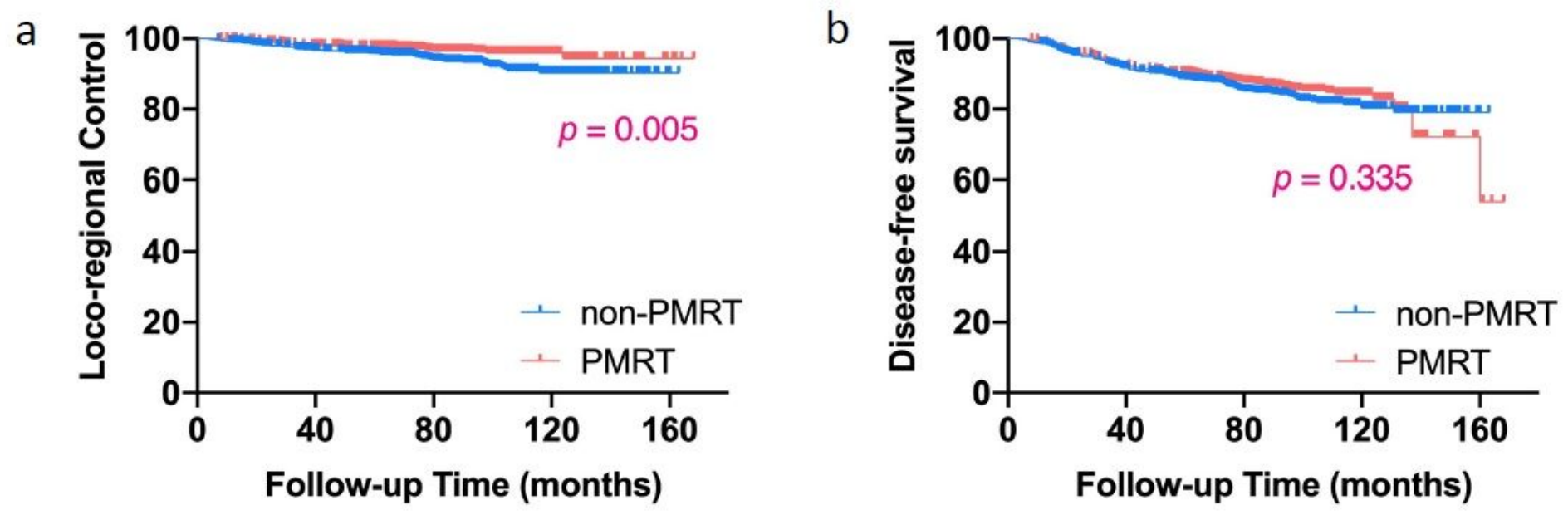

Figure 1

Locoregional control (a) and disease-free survival (b) of patients with or without PMRT for the entire cohort Abbreviations: PMRT = postmastectomy radiotherapy. 
a

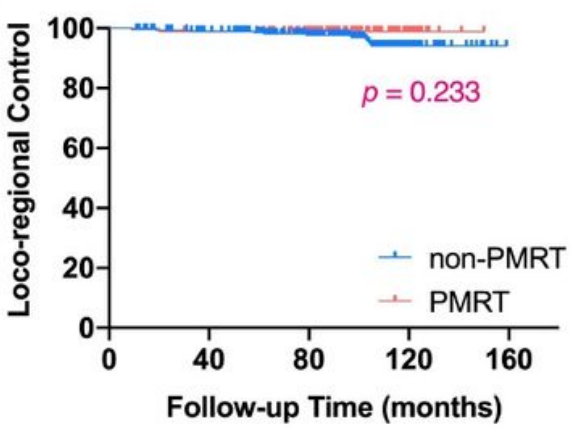

d

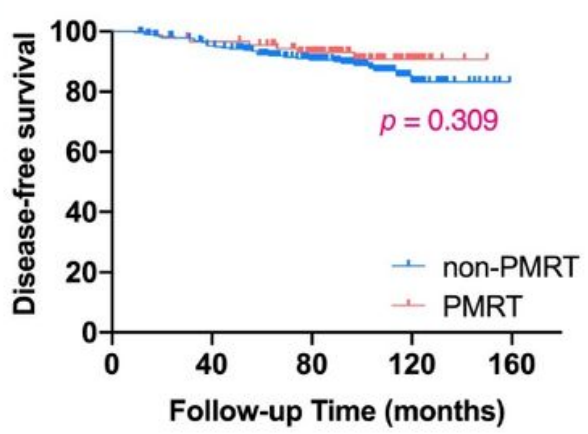

b

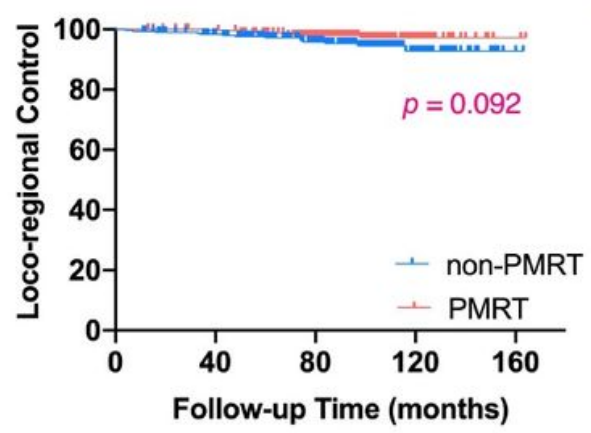

e

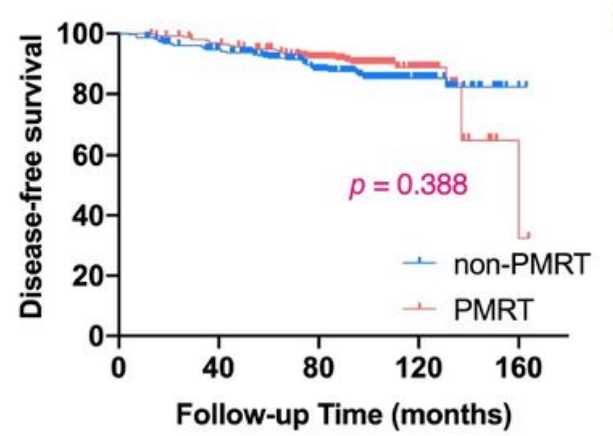

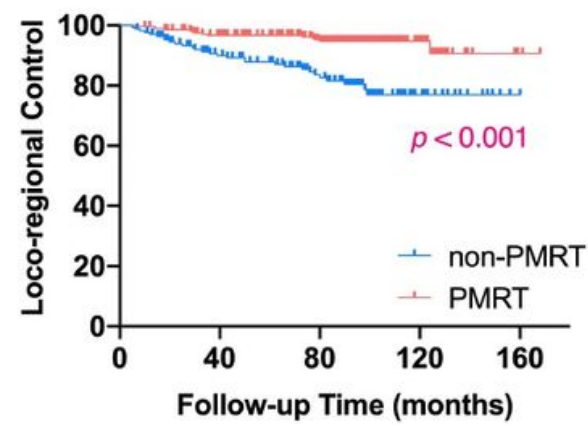

f

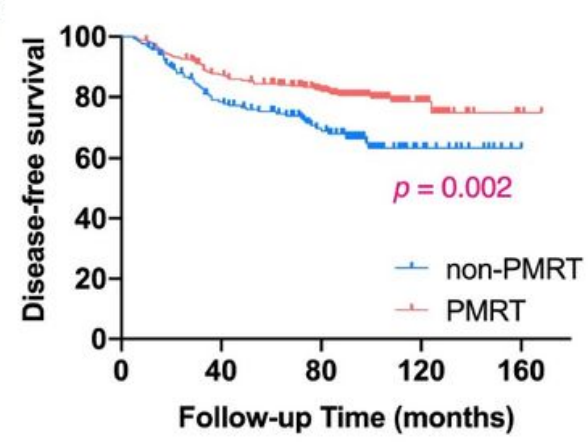

Figure 2

Kaplan-Meier analyses of locoregional control and disease-free survival stratified by recurrence risk. (a, d) low-risk group (no risk factor); (b, e) intermediate-risk group (1 risk factor); (c, f) high-risk group (2-4 risk factors) $\square$ Abbreviations: $\mathrm{PMRT}=$ postmastectomy radiotherapy. 